\title{
Successful immune checkpoint blockade in a patient with advanced stage microsatellite-unstable biliary tract cancer
}

\author{
Elena Czink, ${ }^{1,2}$ Matthias Kloor, ${ }^{3}$ Benjamin Goeppert, ${ }^{2,4}$ Stefan Fröhling, ${ }^{1,6,7}$ \\ Sebastian Uhrig, ${ }^{8}$ Tim F. Weber, ${ }^{9}$ Jörn Meinel, ${ }^{10}$ Christian Sutter, ${ }^{11}$ \\ Karl Heinz Weiss, ${ }^{2,5}$ Peter Schirmacher, ${ }^{2,4}$ Magnus von Knebel Doeberitz, ${ }^{3}$ \\ Dirk Jäger, ${ }^{1,2}$ and Christoph Springfeld ${ }^{1,2}$
}

\begin{abstract}
${ }^{1}$ Department of Medical Oncology, National Center for Tumor Diseases, University Hospital Heidelberg, 69120 Heidelberg, Germany; ${ }^{2}$ Liver Cancer Center Heidelberg, 69120 Heidelberg, Germany; ${ }^{3}$ Department of Applied Tumor Biology, Institute of Pathology, 69120 Heidelberg, Germany; ${ }^{4}$ Institute of Pathology, University Hospital Heidelberg, 69120 Heidelberg, Germany; ${ }^{5}$ Department of Gastroenterology, University Hospital Heidelberg, 69120 Heidelberg, Germany; ${ }^{6}$ Department of Translational Oncology, National Center for Tumor Diseases and German Cancer Research Center, 69120 Heidelberg, Germany; ${ }^{7}$ German Cancer Consortium, 69120 Heidelberg, Germany; ${ }^{8}$ Division of Applied Bioinformatics, German Cancer Research Center, 69120 Heidelberg, Germany; ${ }^{9}$ Department of Diagnostic and Interventional Radiology, University Hospital Heidelberg, 69120 Heidelberg, Germany; ${ }^{10}$ Institute of Pathology, University Hospital Carl Gustav Carus at the Technical University of Dresden, 01307 Dresden, Germany; ${ }^{11}$ Institute of Human Genetics, University Hospital Heidelberg, 69120 Heidelberg, Germany
\end{abstract}

Corresponding author: elena. czink@med.uni-heidelberg.de

(C) 2017 Czink et al. This article is distributed under the terms of the Creative Commons Attribution-NonCommercial License, which permits reuse and redistribution, except for commercial purposes, provided that the original author and source are credited.

Ontology terms: biliary tract neoplasm; neoplasm of the gastrointestinal tract

Published by Cold Spring Harbor Laboratory Press

doi: 10.1101/mcs.a001974
Abstract Cancers acquire multiple somatic mutations that can lead to the generation of immunogenic mutation-induced neoantigens. These neoantigens can be recognized by the host's immune system. However, continuous stimulation of immune cells against tumor antigens can lead to immune cell exhaustion, which allows uncontrolled outgrowth of tumor cells. Recently, immune checkpoint inhibitors have emerged as a novel approach to overcome immune cell exhaustion and reactivate antitumor immune responses. In particular, antibodies blocking the exhaustion-mediating programmed death receptor (PD-1)/ programmed death receptor ligand (PD-L1) pathway have shown clinical efficacy. The effects were particularly pronounced in tumors with DNA mismatch repair (MMR) deficiency and a high mutational load, which typically occur in the colon and endometrium. Here, we report on a 24-yr-old woman diagnosed with extrahepatic cholangiocarcinoma who showed strong and durable response to the immune checkpoint inhibitor pembrolizumab, although treatment was initiated at an advanced stage of disease. The patient's tumor displayed DNA MMR deficiency and microsatellite instability (MSI) but lacked other features commonly discussed as predictors of response toward checkpoint blockade, such as PD-L1 expression or dense infiltration with cytotoxic T cells. Notably, high levels of HLA class I and II antigen expression were detected in the tumor, suggesting a potential causal relation between functionality of the tumor's antigen presentation machinery and the success of immune checkpoint blockade. We suggest determining MSI status in combination with HLA class I and II antigen expression in tumors potentially eligible for immune checkpoint blockade even in the absence of conventional markers predictive for anti-PD-1/PD-L1 therapy and in entities not commonly linked to the MSI phenotype. Further studies are required to determine the value of these markers for predicting the success of immune checkpoint blockade.

[Supplemental material is available for this article.] 


\section{INTRODUCTION}

With the emergence of novel immunotherapies and the encouraging response rates that were demonstrated for checkpoint inhibitors in melanoma patients (Hodi et al. 2010; Topalian et al. 2012; Wolchok et al. 2013), PD-1 and PD-L1 antibodies are in clinical trials for a wide range of malignancies. However, only $30 \%$ of the patients show long-term benefit from this novel immunotherapeutic strategy (Topalian et al. 2012). Hence, this has stimulated interest in characterizing predictors of treatment response to PD-1/PD-L1-guided therapy as well as understanding the escape mechanisms of nonresponding patients. Le et al. demonstrated in a phase II trial that mismatch repair (MMR) deficiency was strongly associated with therapy response to PD-1 blockade in colorectal cancer (CRC) patients as well as a non-CRC cohort (Le et al. 2015). MMR deficiency leads to a typical mutational signature (Alexandrov et al. 2013) and an exceptionally high mutational load-specifically, the accumulation of numerous insertion/deletion mutations affecting microsatellites, a phenotype termed microsatellite instability (MSI). MSI-related mutations of microsatellites located in gene-encoding regions result in the generation of a large number of highly immunogenic frameshift peptide neoantigens (Schwitalle et al. 2008; Kloor and von Knebel Doeberitz 2016). The high number of these MSI-induced frameshift neoantigens explains the pronounced antitumor immune responses and the success of immune checkpoint blockade in these patients. MSI cancers can either develop as sporadic tumors or as hereditary tumors in the context of Lynch syndrome, the most common inherited cancer predisposition syndrome caused by germline mutations of MMR genes. The MSI phenotype is most frequently found in colorectal and endometrial cancers but also occurs in a variety of other malignancies at a lower frequency (Hause et al. 2016). Here we report on a patient with biliary tract cancer, a tumor type rarely displaying MSI (Momoi et al. 2001; Isa et al. 2002; Koo et al. 2003; Liengswangwong et al. 2006), who after extensive pretreatment responded well to PD-1 checkpoint blockade. MSI analysis may open new therapeutic options after standard treatment failure.

\section{RESULTS}

A 24-yr-old female patient was referred in April 2014 for abdominal pain in the right epigastrium. Imaging studies revealed multiple liver lesions up to $5 \mathrm{~cm}$ in diameter and a tumorous mass at the common bile duct, suspicious for extrahepatic cholangiocarcinoma. Histology of one of the liver lesions showed a poorly differentiated carcinoma with an intestinal phenotype and high positivity of CDX-2 and CK7 but negativity for CK20. The tumor markers CA19-9 and CEA were elevated with $979 \mathrm{U} / \mathrm{ml}$ and $99 \mu \mathrm{g} / \mathrm{l}$, respectively. Computed tomography of the lungs showed suspicious pulmonary nodules. Endoscopic workup of the esophagus and stomach as well as the colon did not reveal a primary, hence the diagnosis of a poorly differentiated adenocarcinoma of unknown origin was established with a differential diagnosis of cholangiocarcinoma of intestinal phenotype. RAS mutation testing was negative, and, because of a lack of surgical options, systemic chemotherapy with oxaliplatin and 5-fluorouracil (FOLFOX-4) as well as panitumumab was initiated. With respect to the young age of the patient and the adenocarcinoid differentiation, the patient was referred to genetic counseling. Further analyses of the tumor tissue via MSI typing and immunohistochemistry revealed high MSI and lack of nuclear expression of MMR proteins MLH1 and PMS2. A possible hereditary origin of the tumor was suggested by the following parameters: young age at diagnosis, no BRAF V600E mutation, and lack of $M L H 1$ promoter methylation in the tumor. However, neither germline mutation analysis nor screening for larger deletions or duplications revealed any pathogenic mutation in the MLH1 gene. Exome sequencing of the tumor confirmed a high mutational load with 239 somatic nucleotide substitutions and 


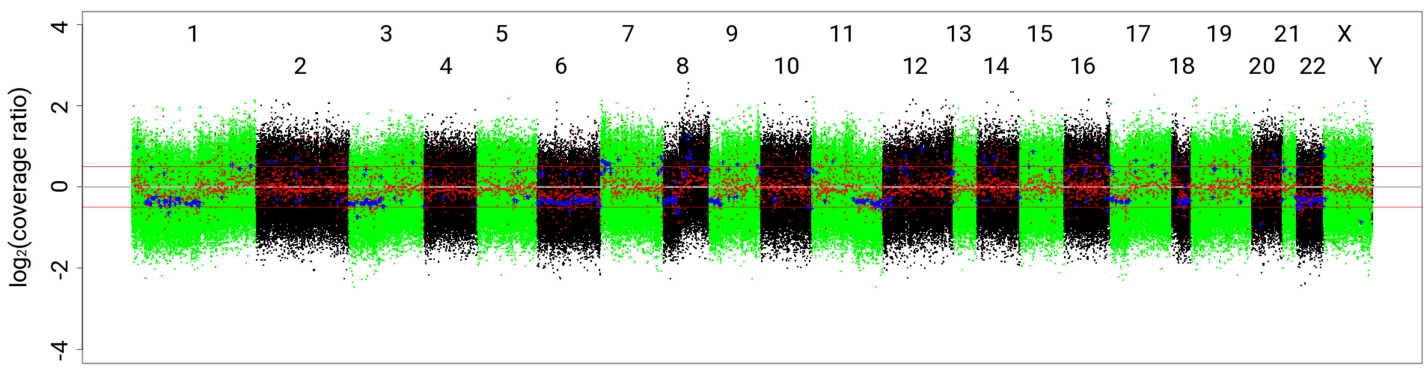

Figure 1. Copy-number variant plot of the patient's tumor material.

48 somatic insertions and deletions but no chromosomal abnormalities (Fig. 1). A variant table stating variant positions is provided in Supplemental Table S1.

The patient responded well to the initial chemotherapy; upon progression after 12 months, she was switched to an irinotecan-based treatment (FOLFIRI) and vascular endothelial growth factor (VEGF) blockade with bevacizumab. With a partial response under this regimen the patient eventually was amenable to extensive liver surgery, allowing for a complete resection of all tumor manifestations. Histopathological analysis revealed a primary tumor mass of $3 \mathrm{~cm}$ in diameter of intestinal differentiation at the cystic duct with high-grade intraepithelial neoplasia in the surrounding epithelium (Fig. 2). Based on these findings, the final diagnosis of cholangiocarcinoma of intestinal differentiation was established. Unfortunately, the patient developed recurrent disease shortly after surgery with multiple new liver lesions. At this point, in an attempt to evaluate further therapeutic options, the patient was included into NCT (National Center for Tumor Diseases) MASTER (Molecularly Aided Stratification for Tumor Eradication Research), a cross-entity program for younger adults with advanced stage cancer. Whole-exome sequencing documented a high mutational load, consistent with the MSI-H status. Reexposure to the FOLFIRI/bevacizumab regimen did not lead to tumor control, so, because of the MSI-H status of the tumor, PD-1 blockade with pembrolizumab (2 mg/ $\mathrm{kg}$ body weight $\mathrm{q} 21$ days) was initiated. After four doses of pembrolizumab, the patient had a partial response of her hepatic lesions on computed tomography (CT) scans (Fig. 3). PD-1 blockade was continued without any autoimmune side effects. Follow-up imaging after

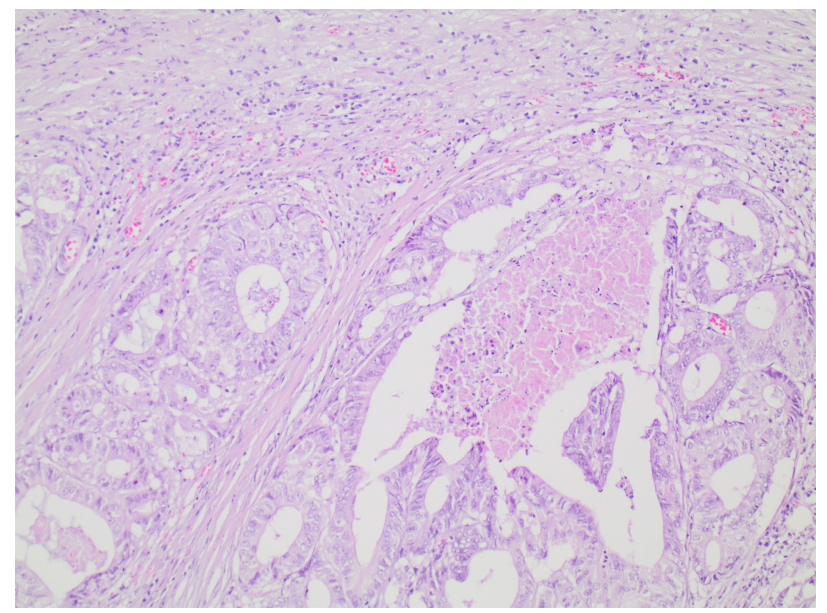

Figure 2. Hematoxylin and eosin (H\&E) staining shows a poorly differentiated adenocarcinoma with a ductular and cribriform growth pattern and focal necrosis (original magnification 200x). 

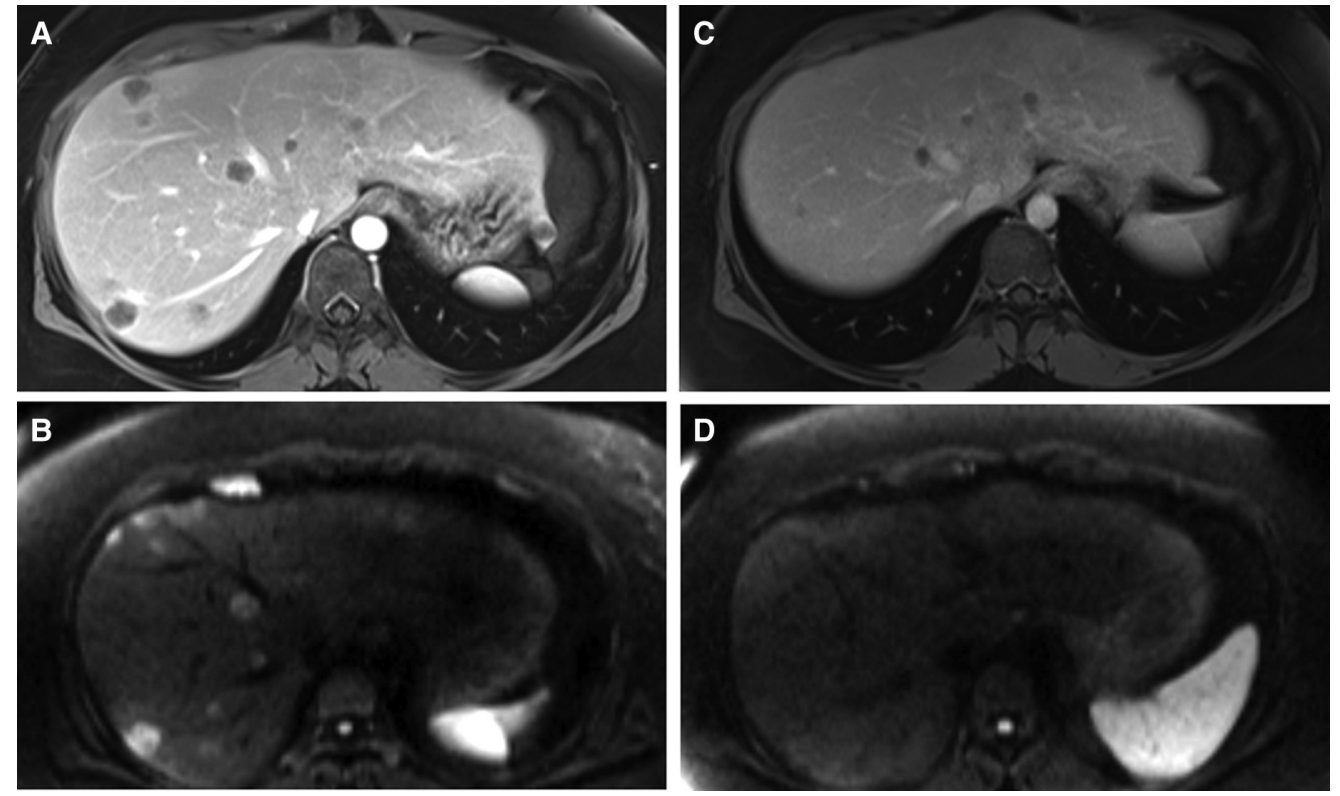

Figure 3. Abdominal magnetic resonance imaging at baseline $(A, B)$ and follow-up $(C, D)$. Contrast-enhanced T1-weighted imaging $(A)$ and diffusion-weighted imaging $(B ; b$-value $=800)$ show multiple hepatic metastases at baseline. The hyperintensity of the lesions on diffusion-weighted imaging represents diffusion restriction due to hypercellularity indicative of active malignancy. At follow-up, contrast-enhanced T1-weighted imaging (C) shows partial response of hepatic metastases according to Immune-related Response Evaluation Criteria In Solid Tumors (irRECIST). The isointensity of the lesions on diffusion-weighted imaging suggests complete regression of hypercellularity.

another four doses demonstrated further reduction of the hepatic tumor burden. Currently, after 13 months of anti-PD-1 treatment the latest CT scan demonstrates stable disease.

\section{DISCUSSION}

Biliary tract cancer (BTC) represents an aggressive disease with a dismal prognosis, hence the development of novel treatment strategies is paramount. There are important differences on both the histopathological and molecular level between intrahepatic cholangiocarcinoma (iCCA), extrahepatic cholangiocarcinoma (eCCA), gallbladder cancer, and ampullary carcinoma (Marsh Rde et al. 2012; Nakamura et al. 2015). In recent years, after extensive sequencing efforts, several potential targeted therapeutic options have evolved for different subgroups of biliary tract cancer (e.g., directed against HER2 in eCCA and gallbladder cancer or IDH1 and FGFR mutations in iCCA). Apart from the molecular differences between iCCA and eCCA, several histological subtypes exist in CCA including an intestinal subtype with tubular glands resembling those of colonic adenocarcinoma, possibly also sharing genetic features of CRC cancers (Rullier et al. 2000).

In the presented case, the intestinal phenotype and the young age of the patient raised the question of a Lynch syndrome-associated cancer. Immunohistochemistry and polymerase chain reaction (PCR)-based tumor analysis revealed MSI-H, accompanied by lack of nuclear MLH1 and PMS2 expression in the tumor, further supporting the hypothesis of Lynch syndrome. However, family history of cancer was negative and no germline mutations were detected in the MLH1 gene, neither in Sanger sequencing nor multiplex ligation-dependent probe amplification (MLPA). On the other hand, the tumor also lacked the typical 
characteristics indicative of sporadic MSI-H colorectal cancers (Parsons et al. 2012), because MLH1 promoter methylation analysis as well as BRAFV600E analysis was negative. However, BRAF V600E mutation has been recently shown to be a rare event in BTC (Goeppert et al. 2014). A "Lynch-like" manifestation caused by two somatic mutations in the MLH1 gene (Buchanan et al. 2014; Mensenkamp et al. 2014; Boland 2016) can also be excluded, because exome sequencing of the tumor did not reveal any somatic pathogenic mutations in the MLH1 gene. In summary, the results on a sporadic or hereditary origin of the tumor remain inconclusive. Therefore, we treat the patient and her family according to the Lynch syndrome guidelines, as a Lynch syndrome (e.g., caused by complex genomic rearrangements not amenable to standard testing [Morak et al. 2014]) cannot be excluded.

However, although hepatocellular carcinoma may rarely be associated with Lynch syndrome as an extracolonic manifestation (Jasperson et al. 2010; Barrow et al. 2013), BTC does not belong to this hereditary cancer syndrome. Data on MSI in BTC is scarce. Momoi et al. (2001) reported an MSI-positive phenotype in four out of 22 samples of intrahepatic cholangiocarcinoma, defined by mononucleotide markers BAT25 and BAT26 and dinucleotide markers such as D2S123. Other groups described a comparable incidence of MSI in BTC (Isa et al. 2002; Koo et al. 2003; Liengswangwong et al. 2006), whereas others found no MSI in their respective cohort (Sessa et al. 2003; Saetta et al. 2004). In the Phase II study of Le et al. (2015) reporting the results of anti-PD-1 antibody therapy in patients with MMR-deficient tumors, four patients with MSI-positive cholangiocarcinoma and ampullary carcinoma were included, but details regarding these cases were not reported. Ampullary carcinoma forms an anatomically and biologically distinct entity, containing tumors that may show biliary, pancreatic, and small bowel features. Extrahepatic cholangiocarcinoma or gallbladder cancer with an intestinal phenotype may share genetic alterations of carcinomas of the lower gastrointestinal tract, potentially including defects of MMR enzymes and MSI.

Clinically, the presented patient showed a partial remission of her hepatic lesions after initiation of PD-1 blockade with pembrolizumab. Le et al. reported an immune-related objective response rate of $40 \%$ in MMR-deficient CRC patients and $0 \%$ in MMR-proficient CRC patients, strongly advocating for a predictive role of MSI for response to PD-1 therapy. However, in microsatellite-unstable colorectal cancers, MMR-deficient tumors that were not associated with Lynch syndrome showed a substantially higher response rate to PD-1 blockade compared with Lynch syndrome patients (100\% to $27 \%, P=0.009$ ) (Le et al. 2015). These findings strongly suggest that more factors are involved in predicting a sustained response to immunotherapy.

Early phase I clinical trials of PD-1 blockade reported an association between clinical response and PD-L1 expression on tumor cells (Topalian et al. 2012; Powles et al. 2014; Weber et al. 2015). With the inhibition of adequate cytotoxic T lymphocyte (CTL) activation through the interaction with coinhibitory molecules like PD-L1, tumor cells avoid immune recognition and destruction, one of many possible immune escape mechanisms. Although patients with PD-L1-negative tumors still demonstrated a clinical benefit in some studies, higher response rates to PD-1 blockade were observed in PD-L1-positive tumors (Spencer et al. 2016). Sabbatino et al. (2016) investigated whether BTC patients mount an immune response to their tumor and checkpoint molecules like PD-L1 are expressed on their tumor cells. Out of 27 iCCA specimens, only in eight tumors was PD-L1 expressed on cancer cells. In their cohort, tumors with a higher T stage expressed more PD-L1 than early-stage iCCAs, suggesting a PD-L1 related immune escape mechanism. In another study, PD-L1 expression within the tumor front was associated with a 60\% decreased survival in iCCA patients (Gani et al. 2016). Our patient, interestingly, stained negative for PD-L1 on tumor cells, only a few leucocytes stained positive for PD-L1 on the tissue samples (Fig. 4), and overall lymphocyte infiltration was sparse.

Down-regulation of HLA class I antigen expression has been suggested as an important mechanism of immune escape in biliary tract cancer (Goeppert et al. 2015; Sabbatino et al. 

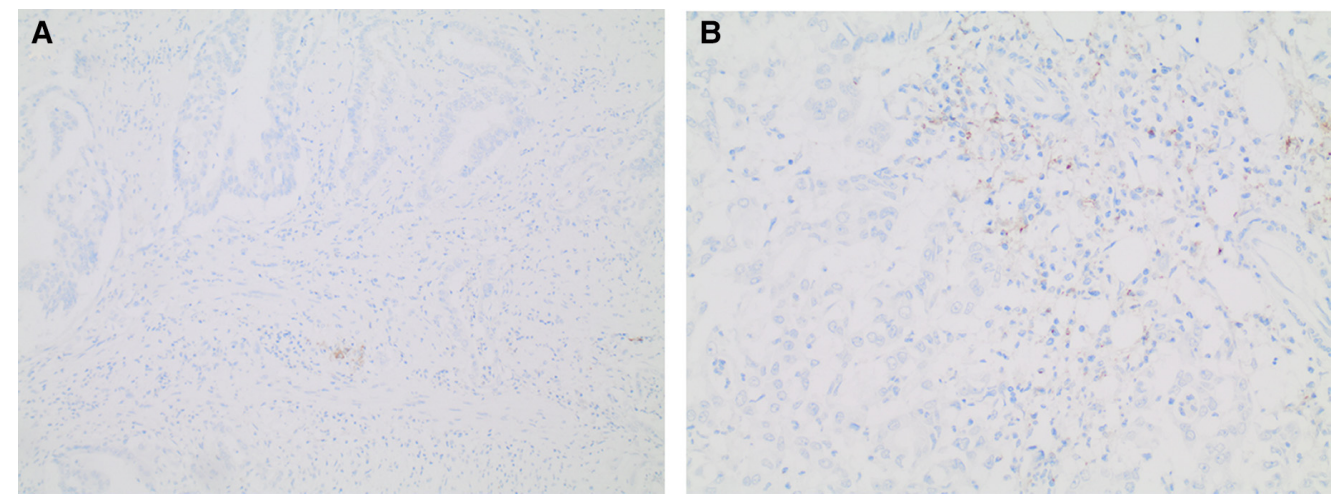

Figure 4. Immunohistochemical stainings of PD-L1 shows negative tumor epithelia and partly positive inflammatory cells in the tumor stroma. Original magnification $100 \times(A)$ and $200 \times(B)$.

2016). Recognition of tumor cells by CTLs is mediated by HLA class I associated antigen presentation, therefore exerting a central role in T-cell-related tumor surveillance. BTC exhibits a wide spectrum of HLA class I expression, with higher expression correlating with lower tumor stages. Importantly, a higher HLA class I expression was also associated with a significantly longer patient survival in a cohort of 302 BTC patients (Goeppert et al. 2015). Interestingly, intraepithelial neoplastic lesions (BillN 3), as seen in our patient, displayed an even higher HLA class I expression in comparison to invasive BTC (Goeppert et al. 2015).

In the presented case, analysis of the HLA expression status of the tumor revealed that all tumor cells showed HLA class I antigen expression on the surface as indicated by homogeneous membrane staining (Fig. 5). This was accompanied by pronounced HLA class Il antigen expression on all tumor cells, as commonly observed in MSI colon cancers (Kataoka et al. 2015). In accordance with these findings, typical mutations that lead to loss of HLA class I or II antigen expression in MSI colorectal cancer, most importantly mutations of $\beta$ 2-microglobulin (B2M) (Kloor et al. 2010), the essential light chain of the HLA class I complex, were not detected in the patient's tumor. These observations suggest a functional tumor antigen presentation machinery, which is a prerequisite for tumor cell recognition by $T$ cells. In melanoma, HLA class II antigen expression has been demonstrated to be required for therapy response toward anti-PD-1 therapy (Johnson et al. 2016). Another study also noted B2M mutations with consecutive loss of surface expression of HLA class I as an acquired resistance mechanism to PD-1 blockade (Zaretsky et al. 2016). Especially within a microsatellite-unstable tumor with a high mutational load, functional HLA class I and II antigen processing and presentation machineries are supposed to be crucial for an adequate stimulation of cognate CTLs.

In summary, we present the case of a young female patient with MSI-H BTC that showed sustained response to anti-PD-1 therapy even at very progressed disease stage. Although MSI is a predictor of therapy response toward immune checkpoint blockade, other potential predictors were lacking in the tumor (e.g., no PD-L1 expression was observed). However, we detected strong HLA class I and II antigen expression. We postulate that HLA class I and II antigen expression status may be positively related to anti-PD-1 therapy success and suggest determining HLA class I and II antigen expression in patients potentially eligible for immune checkpoint modulator therapy. Notably, even in tumors not commonly attributed to a MSI phenotype such as BTC, the combination of MSI-H and strong HLA class I and II antigen expression may be a specific predictor of response toward immune checkpoint modulator.

In addition to the evolving targeted therapies for subgroups of BTC patients, checkpoint inhibition may represent a new option in MSI-H cases. A better understanding of the 

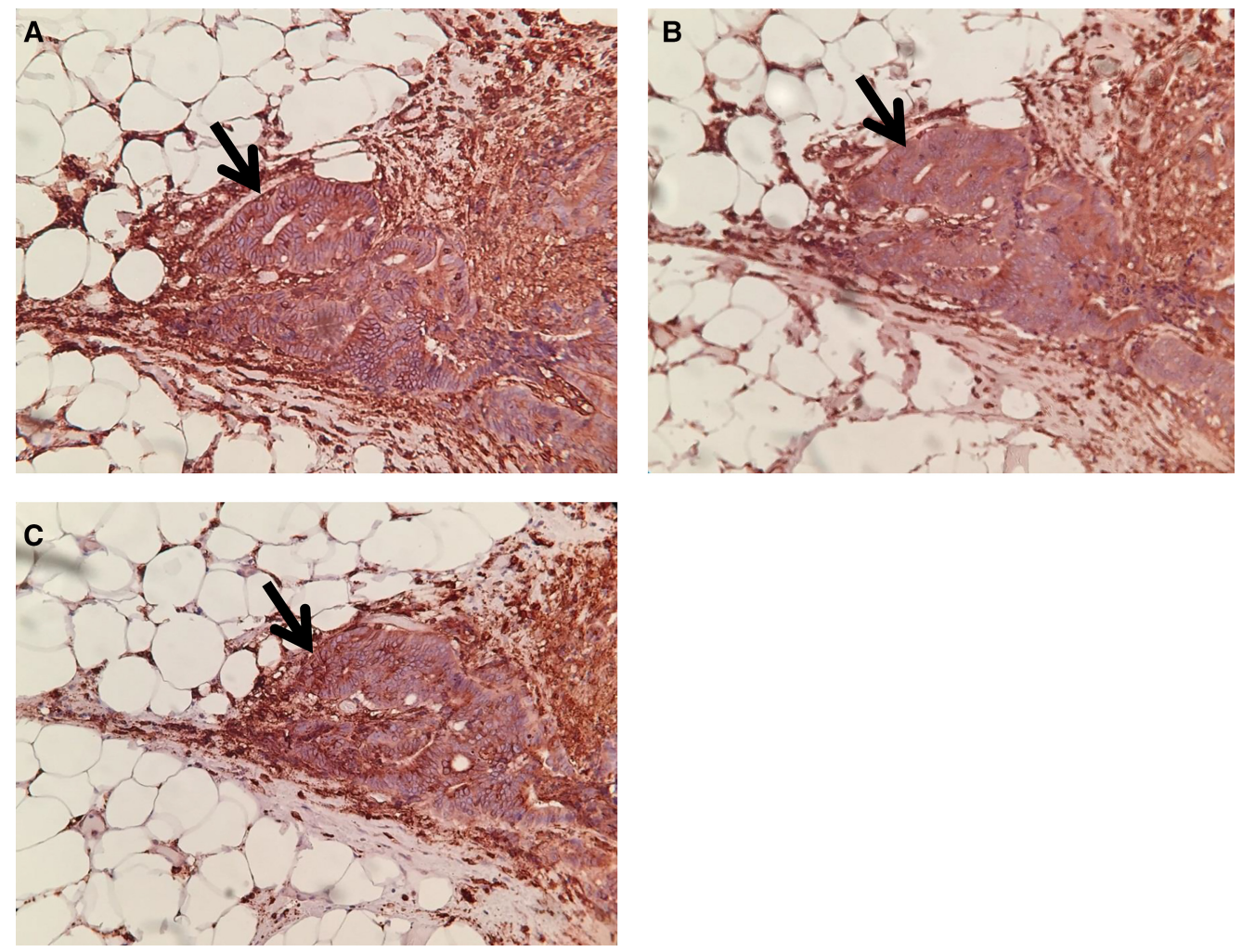

Figure 5. Immunohistochemical stainings of HLA class I $(A, B)$ and HLA class II $(C)$ antigens. Tumor cells display strong membrane staining of HLA class I antigens (heavy chains stained by mAb HC-10, light chain B2M stained by mAb L368; kind gift of Prof. Soldano Ferrone, Boston, USA) and of HLA class II antigens (mAb LGII 6.12-14; S. Ferrone). All pictures are taken with $200 \times$ original magnification. Tumor regions are marked by arrows.

resistance mechanisms against immunotherapy in BTC may lead to increased treatment options for patients with BTC.

\section{METHODS}

DNA Extraction and Microsatellite Instability Analysis

Extraction of genomic DNA was performed after manual microdissection using the QIAGEN DNA Blood and Tissue Kit (QIAGEN) according to the manufacturer's recommendation. DNA content was measured using a NanoDrop (Thermo Scientific). MSI typing was performed using the Bethesda marker panel (Boland et al. 1998) and CAT25 as described previously (Findeisen et al. 2005). High-level MSI (MSI-H) was scored if two or more markers showed MSI.

\section{Immunohistochemistry}

Immunohistochemistry was done on 2- $\mathrm{mm}$ paraffin sections. Briefly, the slides were pretreated by boiling for $15 \mathrm{~min}$ in a microwave oven in citrate buffer (pH 6). First antibodies specific for MMR proteins and PD-L1 were as follows: MSH2 (clone FE11, dilution 1:100; Calbiochem), MLH1 (clone G168-15, dilution 1:100; BD Pharmingen), MSH6 (1:100; clone EP49; Novus Biologicals), PMS2 (1.50; clone A16-4; BD Pharmingen) and PD-L1 (clone 
COLD SPRING HARBOR Molecular Case Studies
Immunotherapy in MSI-H biliary tract cancer
SP263; Ventana Benchmark Ultra, Tuscon). Visualization was carried out following a standard avidin-biotin method (ABC; Vectastain).

\section{Analysis of Germline MLH1 Status}

DNA isolated from blood samples was used for determining the germline allelic MLH1 status by Sanger sequencing. In addition, MLPA was performed using the SALSA MLPA kits P003 MLH1/MSH2 probemix (MRC Holland). Amplified products were visualized on an ABI31 30x automated sequencer (Applied Biosystems/ThermoFisher). Quantification of peak areas was performed using Sequence Pilot software (JSI Medical Systems) according to manufacturer's instructions. Primer sequences are given in the Supplemental Table S2.

\section{Isolation of Analytes}

Tissue samples were provided by the NCT Heidelberg Tissue Bank in accordance with its regulations and after approval by the Ethics Committee of Heidelberg University. DNA from the tumor specimen and the blood sample were isolated using the AllPrep DNA/ RNA/Protein Mini Kit (QIAGEN), followed by quality control and quantification using a Qubit 2.0 Fluorometer (Life Technologies), a 2200 TapeStation system (Agilent), and a 2100 Bioanalyzer system (Agilent).

\section{Whole-Exome Sequencing}

Exome capturing was performed using SureSelect Human All Exon V5+UTRs in-solution capture reagents (Agilent). Briefly, $1.5 \mu \mathrm{g}$ genomic DNA were fragmented to 150-200 bp (paired-end) insert size with a Covaris S2 device (Woburn), and 250 ng of Illumina adapter-containing libraries were hybridized with exome baits at $65^{\circ} \mathrm{C}$ for $16 \mathrm{~h}$. Paired-end sequencing (101 bp) was carried out with a HiSeq 2500 instrument (Illumina) in rapid mode. (See Table 1.)

\section{Mapping and Analysis of Whole-Exome Sequencing Data}

Reads were mapped to the 1000 Genomes Phase 2 assembly of the human reference genome (National Center for Biotechnology Information [NCBI] build 37.1) using BurrowsWheeler alignment (BWA) (version 0.6.2) with default parameters and maximum insert size set to $1000 \mathrm{bp}$ (Li and Durbin 2009). BAM files were sorted with SAMtools (version 0.1.19) (Li et al. 2009), and duplicates were marked with Picard tools (version 1.125). Average target coverage was $127 \times$ for the tumor and $95 \times$ for the control. In both, $>70 \%$ of the targets had a coverage of at least $50 \times$. For the detection of single-nucleotide variants, we applied our inhouse analysis pipeline based on SAMtools mpileup and bcftools with parameter adjustments to allow for calling of somatic variants with heuristic filtering as previously described (Jones et al. 2012, 2013; Yaktapour et al. 2014). After annotation with RefSeq (version September 2013) using ANNOVAR (Wang et al. 2010), somatic, nonsilent coding variants of high confidence were selected. Short insertions and deletions (indels) were identified using Platypus (version 0.5.2; parameters genlndels=1, genSNPs=0, ploidy=2, nlndividuals=2) by providing the tumor and control BAM files (Rimmer et al. 2014). To be of high confidence, somatic calls (control genotype 0/0) were required to either have the Platypus filter flag PASS

Table 1. Whole-exome sequencing coverage metrics

\begin{tabular}{lcccc}
\hline Sample & Total reads & $\begin{array}{c}\text { Percentage of } \\
\text { reads aligned }\end{array}$ & $\begin{array}{c}\text { Percentage of } \\
\text { duplicate reads }\end{array}$ & $\begin{array}{c}\text { Average on-target } \\
\text { read coverage }\end{array}$ \\
\hline Buffy coat & $112,730,942$ & 97.64 & 4.38 & 94.87 \\
Tumor & $163,583,204$ & 95.11 & 13.18 & 127.68 \\
\hline
\end{tabular}


Competing Interest Statement The authors have declared no competing interest.

\section{Referees}

Nelson S. Yee

Anonymous

Received April 7, 2017; accepted in revised form June 6, 2017. or pass custom filters allowing for low variant frequency using a scoring scheme. Candidates with the badReads flag, alleleBias, or strandBias if the variant allele frequency was $<10 \%$, or more than two of the remaining flags were discarded. Additionally, combinations of Platypus non-PASS filter flags, bad quality values, low genotype quality, very low variant counts in the tumor, and presence of variant reads in the control were not tolerated. Indels were annotated with ANNOVAR, and somatic high-confidence indels falling into a coding sequence or splice site were extracted.

Copy-number variants (CNVs) were analyzed by read depth plots and an in-house pipeline using the VarScan2 copynumber and copyCaller modules

Regions were filtered for unmappable genomic stretches, merged by requiring at least 70 markers per called copy-number event and annotated with RefSeq genes using BEDTools (Koboldt et al. 2012).

A table on sequencing coverage is provided in Supplemental Table S3.

\section{ADDITONAL INFORMATION}

\section{Data Deposition and Access}

WES data derived from the patient's tumor tissue were deposited at the European Genomephenome Archive (EGA; https://www.ebi.ac.uk/ega/) under the accession number EGAS00001002441.

\section{Ethics Statement}

All tumor sample and germline genetic analyses under the suspicion of Lynch syndrome were performed in accordance with the German Gene Diagnosis Act. The patient gave written consent for MSI analysis and further immunohistochemical studies. Patient tissue samples from the hepatectomy specimen were collected from the NCT Heidelberg Tissue Bank after written informed consent was obtained under NCT MASTER protocol (S-206/ 2011, approved by the Ethics Committee of Heidelberg University). This protocol covers all aspects relevant to clinical cancer genome sequencing as well as further research and publication. This study was conducted in accordance with the Declaration of Helsinki.

\section{Acknowledgments}

We thank the Human Genetics Institute Heidelberg, the Institute of Pathology Heidelberg, and the Deutsches Krebsforschungszentrum (DKFZ)-HIPO and NCT Precision Oncology Program (POP) Sample Processing Laboratory for technical support and expertise.

\section{Author Contributions}

E.C., M.K., B.G., T.F.W., and C.S. collected and interpreted patient data and were involved in clinical management. M.K., C.S., S.U., and S.F. contributed to data analysis and interpretation. E.C., M.K., B.G., S.F., S.U., T.F.W., J.M., C.S., K.H.W., P.S., M.v.K.D., D.J., and C.S. wrote this manuscript.

\section{Funding}

This work was supported by grant H021 from DKFZ-HIPO (S.F.).

\section{REFERENCES}

Alexandrov LB, Nik-Zainal S, Wedge DC, Aparicio SA, Behjati S, Biankin AV, Bignell GR, Bolli N, Borg A, Børresen-Dale AL, et al. 2013. Signatures of mutational processes in human cancer. Nature 500: 415-421. 
Barrow E, Hill J, Evans DG. 2013. Cancer risk in Lynch syndrome. Fam Cancer 12: 229-240.

Boland CR. 2016. Recent discoveries in the molecular genetics of Lynch syndrome. Fam Cancer 15: 395-403.

Boland CR, Thibodeau SN, Hamilton SR, Sidransky D, Eshleman JR, Burt RW, Meltzer SJ, Rodriguez-Bigas MA, Fodde R, Ranzani GN, et al. 1998. A National Cancer Institute Workshop on Microsatellite Instability for cancer detection and familial predisposition: development of international criteria for the determination of microsatellite instability in colorectal cancer. Cancer Res 58: 5248-5257.

Buchanan DD, Rosty C, Clendenning M, Spurdle AB, Win AK. 2014. Clinical problems of colorectal cancer and endometrial cancer cases with unknown cause of tumor mismatch repair deficiency (suspected Lynch syndrome). Appl Clin Genet 7: 183-193.

Findeisen P, Kloor M, Merx S, Sutter C, Woerner SM, Dostmann N, Benner A, Dondog B, Pawlita M, Dippold W, et al. 2005. T25 repeat in the $3^{\prime}$ untranslated region of the CASP2 gene: a sensitive and specific marker for microsatellite instability in colorectal cancer. Cancer Res 65: 8072-8078.

Gani F, Nagarajan N, Kim Y, Zhu Q, Luan L, Bhaijjee F, Anders RA, Pawlik TM. 2016. Program death 1 immune checkpoint and tumor microenvironment: implications for patients with intrahepatic cholangiocarcinoma. Ann Surg Oncol 23: 2610-2617.

Goeppert B, Frauenschuh L, Renner M, Roessler S, Stenzinger A, Klauschen F, Warth A, Vogel MN, Mehrabi A Hafezi M, et al. 2014. BRAF V600E-specific immunohistochemistry reveals low mutation rates in biliary tract cancer and restriction to intrahepatic cholangiocarcinoma. Mod Pathol 27: 1028-1034.

Goeppert B, Frauenschuh L, Zucknick M, Roessler S, Mehrabi A, Hafezi M, Stenzinger A, Warth A, Pathil A, Renner M, et al. 2015. Major histocompatibility complex class I expression impacts on patient survival and type and density of immune cells in biliary tract cancer. Br J Cancer 113: 1343-1349.

Hause RJ, Pritchard CC, Shendure J, Salipante SJ. 2016. Classification and characterization of microsatellite instability across 18 cancer types. Nat Med 22: 1342-1350.

Hodi FS, O'Day SJ, McDermott DF, Weber RW, Sosman JA, Haanen JB, Gonzalez R, Robert C, Schadendorf D, Hassel JC, et al. 2010. Improved survival with ipilimumab in patients with metastatic melanoma. N Engl J Med 363: 711-723.

Isa T, Tomita S, Nakachi A, Miyazato H, Shimoji H, Kusano T, Muto Y, Furukawa M. 2002. Analysis of microsatellite instability, K-ras gene mutation and p53 protein overexpression in intrahepatic cholangiocarcinoma. Hepatogastroenterology 49: 604-608.

Jasperson KW, Tuohy TM, Neklason DW, Burt RW. 2010. Hereditary and familial colon cancer. Gastroenterology 138: 2044-2058.

Johnson DB, Estrada MV, Salgado R, Sanchez V, Doxie DB, Opalenik SR, Vilgelm AE, Feld E, Johnson AS, Greenplate AR, et al. 2016. Melanoma-specific MHC-II expression represents a tumour-autonomous phenotype and predicts response to anti-PD-1/PD-L1 therapy. Nat Commun 7: 10582.

Jones DT, Jäger N, Kool M, Zichner T, Hutter B, Sultan M, Cho YJ, Pugh TJ, Hovestadt V, Stütz AM, et al. 2012. Dissecting the genomic complexity underlying medulloblastoma. Nature 488: 100-105.

Jones DT, Hutter B, Jäger N, Korshunov A, Kool M, Warnatz HJ, Zichner T, Lambert SR, Ryzhova M, Quang DA, et al. 2013. Recurrent somatic alterations of FGFR1 and NTRK2 in pilocytic astrocytoma. Nat Genet 45: 927-932.

Kataoka K, Nagata Y, Kitanaka A, Shiraishi Y, Shimamura T, Yasunaga J, Totoki Y, Chiba K, Sato-Otsubo A, Nagae G, et al. 2015. Integrated molecular analysis of adult T cell leukemia/lymphoma. Nat Genet 47: 1304-1315.

Kloor M, von Knebel Doeberitz M. 2016. The immune biology of microsatellite-unstable cancer. Trends Cancer 2: 121-133.

Kloor M, Michel S, von Knebel Doeberitz M. 2010. Immune evasion of microsatellite unstable colorectal cancers. Int J Cancer 127: 1001-1010.

Koboldt DC, Zhang Q, Larson DE, Shen D, McLellan MD, Lin L, Miller CA, Mardis ER, Ding L, Wilson RK. 2012. VarScan 2: somatic mutation and copy number alteration discovery in cancer by exome sequencing. Genome Res 22: 568-576.

Koo SH, Ihm CH, Kwon KC, Lee JS, Park JW, Kim JW. 2003. Microsatellite alterations in hepatocellular carcinoma and intrahepatic cholangiocarcinoma. Cancer Genet Cytogenet 146: 139-144.

Le DT, Uram JN, Wang H, Bartlett BR, Kemberling H, Eyring AD, Skora AD, Luber BS, Azad NS, Laheru D, et al. 2015. PD-1 blockade in tumors with mismatch-repair deficiency. N Engl J Med 372: 2509-2520.

$\mathrm{Li} \mathrm{H}$, Durbin R. 2009. Fast and accurate short read alignment with Burrows-Wheeler transform. Bioinformatics 25: $1754-1760$.

Li H, Handsaker B, Wysoker A, Fennell T, Ruan J, Homer N, Marth G, Abecasis G, Durbin R; 1000 Genome Project Data Processing Subgroup. 2009. The Sequence Alignment/Map format and SAMtools. Bioinformatics 25: 2078-2079.

Liengswangwong U, Karalak A, Morishita Y, Noguchi M, Khuhaprema T, Srivatanakul P, Miwa M. 2006. Immunohistochemical expression of mismatch repair genes: a screening tool for predicting mutator 
phenotype in liver fluke infection-associated intrahepatic cholangiocarcinoma. World J Gastroenterol 12: 3740-3745.

Marsh Rde W, Alonzo M, Bajaj S, Baker M, Elton E, Farrell TA, Gore RM, Hall C, Nowak J, Roy H, et al. 2012. Comprehensive review of the diagnosis and treatment of biliary tract cancer 2012. Part I: diagnosisclinical staging and pathology. J Surg Oncol 106: 332-338.

Mensenkamp AR, Vogelaar IP, van Zelst-Stams WA, Goossens M, Ouchene H, Hendriks-Cornelissen SJ, Kwint MP, Hoogerbrugge N, Nagtegaal ID, Ligtenberg MJ. 2014. Somatic mutations in MLH1 and MSH2 are a frequent cause of mismatch-repair deficiency in Lynch syndrome-like tumors. Gastroenterology 146: 643-646 e648.

Momoi H, Itoh T, Nozaki Y, Arima Y, Okabe H, Satoh S, Toda Y, Sakai E, Nakagawara K, Flemming P, et al. 2001. Microsatellite instability and alternative genetic pathway in intrahepatic cholangiocarcinoma. $J$ Hepatol 35: 235-244.

Morak M, Heidenreich B, Keller G, Hampel H, Laner A, de la Chapelle A, Holinski-Feder E. 2014. Biallelic MUTYH mutations can mimic Lynch syndrome. Eur J Hum Genet 22: 1334-1337.

Nakamura H, Arai Y, Totoki Y, Shirota T, Elzawahry A, Kato M, Hama N, Hosoda F, Urushidate T, Ohashi S, et al. 2015. Genomic spectra of biliary tract cancer. Nat Genet 47: 1003-1010.

Parsons MT, Buchanan DD, Thompson B, Young JP, Spurdle AB. 2012. Correlation of tumour BRAF mutations and $\mathrm{MLH} 1$ methylation with germline mismatch repair (MMR) gene mutation status: a literature review assessing utility of tumour features for MMR variant classification. J Med Genet 49: 151-157.

Powles T, Eder JP, Fine GD, Braiteh FS, Loriot Y, Cruz C, Bellmunt J, Burris HA, Petrylak DP, Teng SL, et al. 2014. MPDL3280A (anti-PD-L1) treatment leads to clinical activity in metastatic bladder cancer. Nature 515: 558-562.

Rimmer A, Phan H, Mathieson I, lqbal Z, Twigg SR, WGS500 Consortium, Wilkie AO, McVean G, Lunter G. 2014. Integrating mapping-, assembly- and haplotype-based approaches for calling variants in clinical sequencing applications. Nat Genet 46: 912-918.

Rullier A, Le Bail B, Fawaz R, Blanc JF, Saric J, Bioulac-Sage P. 2000. Cytokeratin 7 and 20 expression in cholangiocarcinomas varies along the biliary tract but still differs from that in colorectal carcinoma metastasis. Am J Surg Pathol 24: 870-876.

Sabbatino F, Villani V, Yearley JH, Deshpande V, Cai L, Konstantinidis IT, Moon C, Nota S, Wang Y, AlSukaini A, et al. 2016. PD-L1 and HLA class I antigen expression and clinical course of the disease in intrahepatic cholangiocarcinoma. Clin Cancer Res 22: 470-478.

Saetta AA, Papanastasiou P, Michalopoulos NV, Gigelou F, Korkolopoulou P, Bei T, Patsouris E. 2004. Mutational analysis of BRAF in gallbladder carcinomas in association with K-ras and p53 mutations and microsatellite instability. Virchows Arch 445: 179-182.

Schwitalle Y, Kloor M, Eiermann S, Linnebacher M, Kienle P, Knaebel HP, Tariverdian M, Benner A, von Knebel Doeberitz M. 2008. Immune response against frameshift-induced neopeptides in HNPCC patients and healthy HNPCC mutation carriers. Gastroenterology 134: 988-997.

Sessa F, Furlan D, Genasetti A, Billo P, Feltri M, Capella C. 2003. Microsatellite instability and p53 expression in gallbladder carcinomas. Diagn Mol Pathol 12: 96-102.

Spencer KR, Wang J, Silk AW, Ganesan S, Kaufman HL, Mehnert JM. 2016. Biomarkers for immunotherapy: current developments and challenges. Am Soc Clin Oncol Educ Book 35: e493-e503.

Topalian SL, Hodi FS, Brahmer JR, Gettinger SN, Smith DC, McDermott DF, Powderly JD, Carvajal RD, Sosman JA, Atkins MB, et al. 2012. Safety, activity, and immune correlates of anti-PD-1 antibody in cancer. N Engl J Med 366: 2443-2454.

Wang K, Li M, Hakonarson H. 2010. ANNOVAR: functional annotation of genetic variants from high-throughput sequencing data. Nucleic Acids Res 38: e164.

Weber JS, D'Angelo SP, Minor D, Hodi FS, Gutzmer R, Neyns B, Hoeller C, Khushalani NI, Miller WH Jr, Lao CD, et al. 2015. Nivolumab versus chemotherapy in patients with advanced melanoma who progressed after anti-CTLA-4 treatment (CheckMate 037): a randomised, controlled, open-label, phase 3 trial. Lancet Oncol 16: 375-384.

Wolchok JD, Kluger H, Callahan MK, Postow MA, Rizvi NA, Lesokhin AM, Segal NH, Ariyan CE, Gordon RA, Reed K, et al. 2013. Nivolumab plus ipilimumab in advanced melanoma. N Engl J Med 369: 122-133.

Yaktapour N, Meiss F, Mastroianni J, Zenz T, Andrlova H, Mathew NR, Claus R, Hutter B, Fröhling S, Brors B, et al. 2014. BRAF inhibitor-associated ERK activation drives development of chronic lymphocytic leukemia. $J$ Clin Invest 124: 5074-5084.

Zaretsky JM, Garcia-Diaz A, Shin DS, Escuin-Ordinas H, Hugo W, Hu-Lieskovan S, Torrejon DY, AbrilRodriguez G, Sandoval S, Barthly L, et al. 2016. Mutations associated with acquired resistance to PD-1 blockade in melanoma. N Engl J Med 375: 819-829. 


\section{COLD SPRING HARBOR Molecular Case Studies}

\section{Successful immune checkpoint blockade in a patient with advanced stage microsatellite-unstable biliary tract cancer}

Elena Czink, Matthias Kloor, Benjamin Goeppert, et al.

Cold Spring Harb Mol Case Stud 2017, 3: a001974 originally published online June 15, 2017 Access the most recent version at doi: $10.1101 / \mathrm{mcs} . a 001974$
Supplementary http://molecularcasestudies.cshlp.org/content/suppl/2017/06/15/mcs.a001974.D Material C1
References This article cites 45 articles, 5 of which can be accessed free at: http://molecularcasestudies.cshlp.org/content/3/5/a001974.full.html\#ref-list-1
License This article is distributed under the terms of the Creative Commons Attribution-NonCommercial License, which permits reuse and redistribution, except for commercial purposes, provided that the original author and source are credited.
Email Alerting Receive free email alerts when new articles cite this article - sign up in the box at the Service top right corner of the article or click here.

